\title{
EDITORIAL
}

\section{Pulmonologists and respiratory intensive care}

\author{
C. Roussos, A. Rossi
}

In several countries, particularly North America, intensive care medicine is closely linked to pulmonology. The curriculum of pulmonologists in training has an obligatory period in the Intensive Care Unit (ICU). In most European countries, pulmonologists do not have any role or have only a very small role in the treatment of severe failure of the lung and thoracic pump, which is their field of specialization. More importantly, in Europe in the 4 yrs of training needed to become a European Community (EC) certified pulmonologist, a period in the ICU is not included. This is to say that a general physician can become a pulmonologist without having been exposed to the complex procedures [1] and problems [2] leading to the diagnosis, monitoring, treatment and management of patients with severe respiratory failure.

The fact that pulmonologists are not exposed to respiratory intensive care problems has important and multifactorial implications. Firstly, fundamental exposure to the pathophysiology and treatment of respiratory diseases is missed. Secondly, early exposure to critical care problems would allow pulmonologists to consider the possibilities of following a career in respiratory intensive care. Thus, in addition to educational benefits, such exposure might open up new horizons for young pulmonologists. In our view, therefore, it is essential that, in the future, an effort should be made to ensure that the curriculum of new pulmonologists includes the experience of "stepdown" or "intermediate" care units or chronic home ventilatory assistance for chronic respiratory failure, which could help to reduce the general ICU cost [3-5]. In these

Correspondence: C. Roussos, Critical Care Department, Evangelismos General Hospital, 45-47 Ipsilandou Str, GK-11521 Athens, Greece. A. Rossi, Ospedale Maggiore di Borgo Trento, Resp. Division, P. le Stefanie, 1, I-37126 Verona, Italy. settings, experienced pneumologists/intensivists have and should play an important role.

The European Respiratory Society (ERS) has a special interest in increasing the involvement of pulmonologists in intensive care medicine. Efforts are being made by the Scientific Assembly of Respiratory Intensive Care to promote this discipline during ERS Congresses, as well as by institution of task forces and interaction with the European Society of Intensive Care Medicine (ESICM) and the American Thoracic Society (ATS). In addition to those efforts, the European Respiratory Journal has created a new series on "Clinical Physiology in Respiratory Intensive Care", which begins in this issue of the Journal. In this series, a number of review articles from internationally recognized experts will be published covering many aspects of the pathophysiology involved in respiratory intensive care medicine, with particular attention to clinical applications.

We hope that this series will be highly educational and will also promote the interest of pulmonologists in intensive care medicine.

\section{References}

1. Hansen-Flaschen J. Pulmonary and critical care procedures. Am J Respir Crit Care Med 1995; 151: 275-277.

2. Lanken PN. Critical care medicine at new crossroads. Am J Respir Crit Care Med 1994; 149: 3-5.

3. Snider GL. Allocation of intensive care. Am J Respir Crit Care Med 1994; 150: 575-580.

4. Zalenski D. Estimating the cost of intensive care. Intensive Care Med 1994; 20: 538-539.

5. Rossi A, Ambrosino N. The need for an intermediate cardiorespiratory unit. Monaldi Arch Chest Dis 1994; 49: 463-465. 